\title{
Smartphone Cognitive Behavioral Therapy as an Adjunct to Pharmacotherapy for Refractory Depression: Randomized Controlled Trial
}

Akio Mantani ${ }^{1}$, MD, PhD; Tadashi Kato ${ }^{2}$, MD; Toshi A Furukawa ${ }^{3}, \mathrm{MD}, \mathrm{PhD}$; Masaru Horikoshi ${ }^{4}, \mathrm{PhD}$; Hissei Imai $^{3}, \mathrm{MD}, \mathrm{PhD}$; Takahiro Hiroe ${ }^{5}, \mathrm{MD}, \mathrm{PhD}$; Bun Chino ${ }^{6}, \mathrm{MD}, \mathrm{PhD}$; Tadashi Funayama ${ }^{7}, \mathrm{MD}, \mathrm{PhD}$; Naohiro Yonemoto $^{8}$, MPH; Qi Zhou ${ }^{9}, \mathrm{PhD} ; \mathrm{Nao}$ Kawanishi $^{10}, \mathrm{PhD}$

${ }^{1}$ Mantani Mental Clinic, Hiroshima, Japan

${ }^{2}$ Aratama Kokorono Clinic, Nagoya, Japan

${ }^{3}$ Department of Health Promotion and Human Behavior, School of Public Health, Graduate School of Medicine, Kyoto University, Kyoto, Japan

${ }^{4}$ National Center of Neurology and Psychiatry, Kodaira, Japan

${ }^{5}$ Waseda Clinic, Kani, Japan

${ }^{6}$ Ginza Taimei Clinic, Tokyo, Japan

${ }^{7}$ Funayama Mental Clinic, Nagoya, Japan

${ }^{8}$ Department of Biostatistics, School of Public Health, Graduate School of Medicine, Kyoto University, Kyoto, Japan

${ }^{9}$ Department of Health Research Methods, Evidence, and Impact, McMaster University, Hamilton, ON, Canada

${ }^{10}$ Advanced Telecommunications Research Institute International, Kyoto, Japan

$\ddagger_{\text {the FLATT Investigators }}$

\section{Corresponding Author:}

Toshi A Furukawa, MD, PhD

Department of Health Promotion and Human Behavior

School of Public Health, Graduate School of Medicine

Kyoto University

Yoshida Konoe-cho

Sakyo-ku

Kyoto, 6068501

Japan

Phone: 81757538941

Fax: 81757534643

Email: furukawa@kuhp.kyoto-u.ac.jp

\section{Related Article:}

This is a corrected version. See correction statement in: http://www.jmir.org/2018/8/e11702/

\section{Abstract}

Background: In the treatment of major depression, antidepressants are effective but not curative. Cognitive behavioral therapy (CBT) is also effective, alone or in combination with pharmacotherapy, but accessibility is a problem.

Objective: The aim is to evaluate the effectiveness of a smartphone CBT app as adjunctive therapy among patients with antidepressant-resistant major depression.

Methods: A multisite, assessor-masked, parallel-group randomized controlled trial was conducted in 20 psychiatric clinics and hospitals in Japan. Participants were eligible if they had a primary diagnosis of major depression and were antidepressant-refractory after taking one or more antidepressants at an adequate dosage for four or more weeks. After a 1-week run-in in which participants started the medication switch and had access to the welcome session of the app, patients were randomized to medication switch alone or to medication switch plus smartphone CBT app via the centralized Web system. The smartphone app, called Kokoro-app ("kokoro" means "mind" in Japanese), included sessions on self-monitoring, behavioral activation, and cognitive restructuring presented by cartoon characters. The primary outcome was depression severity as assessed by masked telephone assessors with the Patient Health Questionnaire-9 (PHQ-9) at week 9. The secondary outcomes included the Beck Depression Inventory-II (BDI-II) and Frequency, Intensity, and Burden of Side Effects Ratings (FIBSER). 
Results: In the total sample $(\mathrm{N}=164), 81$ participants were allocated to the smartphone CBT in addition to medication change and 83 to medication change alone. In the former group, all but one participant $(80 / 81,99 \%)$ completed at least half, and $71(88 \%)$ completed at least six of eight sessions. In the intention-to-treat analysis, patients allocated the CBT app scored 2.48 points $(95 \%$ CI 1.23-3.72, $P<.001$; standardized mean difference 0.40) lower on PHQ-9 than the control at week 9. The former group also scored 4.1 points $(95 \%$ CI $1.5-6.6, P=.002)$ lower on BDI-II and 0.76 points $(95 \% \mathrm{CI}-0.05$ to $1.58, P=.07)$ lower on FIBSER. In the per-protocol sample (comfortable with the smartphone app, still symptomatic, and adherent to medication with mild or less side effects after run-in), the intervention group ( $\mathrm{n}=60)$ scored 1.72 points (95\% CI 0.25-3.18, $P=.02)$ lower on PHQ-9, 3.2 points $(95 \% \mathrm{CI}-0.01$ to $6.3, P=.05$ ) lower on BDI-II, and 0.75 points $(95 \% \mathrm{CI} 0.03-1.47, P=.04$ ) lower on FIBSER than the control $(n=57)$. The treatment benefits were maintained up to week 17.

Conclusions: This is the first study to demonstrate the effectiveness of a smartphone CBT in the treatment of clinically diagnosed depression. Given the merits of the mobile mental health intervention, including accessibility, affordability, quality control, and effectiveness, it is clinically worthwhile to consider adjunctive use of a smartphone CBT app when treating patients with antidepressant-resistant depression. Research into its effectiveness in wider clinical contexts is warranted.

Trial Registration: Japanese Clinical Trials Registry UMIN CTR 000013693; https://upload.umin.ac.jp/cgi-open-bin/ctr_e/ ctr_view.cgi?recptno=R000015984 (Archived by WebCite at http://www.webcitation.org/6u6pxVwik)

(J Med Internet Res 2017;19(11):e373) doi: 10.2196/jmir.8602

\section{KEYWORDS}

major depressive disorder; pharmacotherapy-resistant depression; mobile phone app; cognitive behavioral therapy; eHealth

\section{Introduction}

Major depression is highly prevalent, debilitating, and costly [1-3]. It is predicted to be the leading cause of premature mortality and disability in high-income countries by 2030 , and the third-leading cause in low- and middle-income countries [4]. Resources for and access to care by those who suffer remain constrained in high-income countries, and severely so in lowto middle-income ones $[5,6]$.

Although antidepressant pharmacotherapy represents the mainstay of treatment of major depression [7], after several weeks of treatment only $50 \%$ show reduction by half or more in their depression severity and only $30 \%$ return to a euthymic state [8]. No standard approach in the management of treatment-refractory depression exists. Guideline recommendations include increasing the dose, switching to another antidepressant, or augmenting treatment with another pharmacological or psychological therapy $[9,10]$.

Cognitive behavioral therapy (CBT) has proved an effective treatment of major depression either alone [11] or in combination with pharmacotherapy $[12,13]$. A standard course of CBT, however, requires 10 to 20 sessions, each lasting 45 to 60 minutes, with an adequately qualified professional. Therefore, its availability is limited everywhere in the world [14,15]. Telephone or videoconference CBT eliminates the burden of in-person visits, but requires approximately equal therapist time and equal competence.

Guided self-help CBT requires minimal to no clinician time and has proved of comparable effectiveness to its face-to-face versions [16]. Information and communication technologies (ICT) for self-help CBT, including computerized stand-alone software and Internet-based webpages, have shown promising results in initial trials [17]. However, one recent pragmatic trial using two widely known online Web-based CBT packages failed to demonstrate added value over usual care in primary care likely because adherence to the program was very low [18].
The dominant modality of ICT is evolving rapidly and the smartphone is now transforming people's lives across the world. In comparison with stand-alone or Internet-connected computers, the smartphone enhances portability and immediacy, making CBT fully accessible and therefore promising new dimensions of guided self-help. Mobile health apps are currently proliferating in the electronic world, with more than 165,000 health apps available online [19-21]. However, few apps have demonstrated quality $[22,23]$ and no randomized controlled trial (RCT) has yet proved the benefits of a smartphone app in comparison with a control condition in the treatment of clinically diagnosed major depressive disorder. Several trials have examined the use of smartphone to monitor symptoms [24-26] or smartphone apps applying the CBT principles, but only among participants recruited from the general population who had reported elevated levels of depressive symptoms on self-reports [27-33]. A few have examined participants with diagnosed major depression, but only against active controls. One pilot RCT compared the original Web-based CBT against its smartphone version in 35 participants with depression confirmed through telephone diagnostic interview [34]. Another group of researchers developed a smartphone app for behavioral activation and tested it against its face-to-face full version [35] or against a mindfulness-based program [36] among 93 and 81 patients, respectively, who were recruited through advertisements in mass media but whose diagnosis of major depression was confirmed through telephone interview. In these studies, results did not differ significantly between the intervention and control groups.

Whether smartphone-based CBT can have any demonstrable value in the treatment of clinically diagnosed major depression is a pressing issue for patients, clinicians, and policy makers around the world $[22,23]$. We have developed and pilot-tested a smartphone app, called Kokoro-app (kokoro means "mind" in Japanese), that is based on a CBT manual with demonstrated effectiveness in remote telephone or group formats in several RCTs either alone [37,38] or in combination with 
antidepressants [39]. This study represents the first RCT to examine adjunctive smartphone-based CBT to medication change among patients with major depression unresponsive to prior antidepressant treatments.

\section{Methods}

\section{Design}

The study was a multisite, assessor-masked, parallel-group RCT with a 1:1 allocation ratio.

\section{Participants}

A detailed description of the study protocol has been reported elsewhere [40] and is attached as Multimedia Appendix 1. The study, which was approved by the ethics committees of the participating centers and registered in the Japanese clinical trials registry (UMIN CTR 000013693), took place in 20 psychiatric clinics and hospitals across Japan between September 2014 and October 2016. Multimedia Appendix 2 provides the complete list of trial sites and investigators.

Eligible participants (1) were aged between 25 and 59 years, (2) had a primary diagnosis of major depressive disorder without psychotic features according to the Diagnostic and Statistical Manual of Mental Disorders (Fifth Edition) [41] as ascertained by using the Primary Care Evaluation of Mental Disorders procedure [42], (3) were antidepressant-resistant, defined as scoring 10 or more on the Beck Depression Inventory-II (BDI-II) $[37,43]$ after taking one or more antidepressants at an adequate dosage for four or more weeks (stage I, II, or III according to the criteria by Thase and Rush [44]), (4) had not been prescribed escitalopram or sertraline, or received CBT or interpersonal therapy for the index episode.

The study psychiatrists introduced the trial to the potentially eligible patients from among the patients they were seeing and invited them to participate voluntarily. After full disclosure of the trial contents and procedures, all participants provided written informed consent. No advertisement through the media was used.

\section{Randomization}

On entry, all participants started switching their antidepressant, had the Kokoro-app installed onto their iPhone, and had access to the welcome session, which mainly aimed at, after a brief description of CBT, training the participants in the use of the smartphone and its speech recognition. (When the participants did not own an iPhone, we lent one to them for the duration of the trial.) After this 1-week run-in, they had a telephone interview with the Patient Health Questionnaire-9 (PHQ-9) and the Frequency, Intensity, and Burden of Side Effects Ratings (FIBSER) and were classified into one of two samples. The per-protocol sample comprised those who had not or only partially responded since week 0 (PHQ-9 scores $\geq 5$ [45] at week 1), who tolerated and adhered to the new antidepressant, and had no problem using the smartphone and Kokoro-app. The auxiliary sample included all others. The total sample (the per-protocol sample plus the auxiliary sample) would answer the real-world question of the value of smartphone CBT among the patients for whom the clinicians would initially consider prescribing the smartphone CBT, including those who would not tolerate the new medication or who may have some difficulty using the smartphone app, whereas the per-protocol sample would answer the question of the value of smartphone CBT under the narrower circumstances (ie, among patients who were able to follow the protocol and for whom the app would be expected to demonstrate its full effects).

After stratification by group, the participants were randomized 1:1 to the combined antidepressant switch plus smartphone CBT arm (intervention arm) or the antidepressant switch alone arm (control arm) using an automated Web program implementing the method of minimization. Therefore, the randomization was concealed. Clinics, number of antidepressants previously prescribed for the index episode ( $\geq 3$ vs $<3$ ), and a total score of the PHQ-9 at week $1(\geq 10$ vs $<10)$ were used as minimization variables.

During the telephone interview for the PHQ-9, if participants reported suicidal ideation for more than half the days or nearly every day of the previous 2 weeks, the interviewer immediately notified the staff in the central office, who then notified the responsible psychiatrist.

\section{Interventions}

The intervention group received both the antidepressant switch and the smartphone CBT, whereas the control group received only the antidepressant switch during the intervention period up to week 9. Details of each intervention are described subsequently.

\section{Antidepressant Switch}

All study participants started switching their antidepressant either to escitalopram (5-10 mg/day) or to sertraline (25-100 $\mathrm{mg} /$ day) at week 0 . The previous antidepressant was tapered off by week 5 . We limited choice of antidepressants to escitalopram and sertraline, which showed a favorable profile in efficacy and acceptability in a previous systematic review [46], to ensure balance in antidepressant treatments during the trial. If the participants did not tolerate escitalopram or sertraline, the physician could revert to the previous antidepressant or start a new one. Only anxiolytics and hypnotics were allowed as coprescribed psychotropics. The frequency of visit was set at least once in four weeks, with additional visits as judged necessary by the study physician.

\section{Smartphone Cognitive Behavioral Therapy}

Kokoro-app is a self-help smartphone app consisting of eight sessions, including one welcome session, two sessions on self-monitoring, two sessions on behavioral activation, two sessions on cognitive restructuring, and an epilog focusing on relapse prevention. In each session, explanation of the principles and skills of CBT is provided in the format of instant messenger exchanges among cartoon characters (Figure 1). In the self-monitoring sessions, patients learn how to monitor their reactions to situations in terms of feelings, thoughts, body reactions, and behaviors and describe them in "mind maps." In the behavioral activation sessions, patients learn to engage in "personal experiments" of small pleasurable actions according to the principle "When your body moves, so does your mind." 
Figure 1. Screenshots from Kokoro-app.

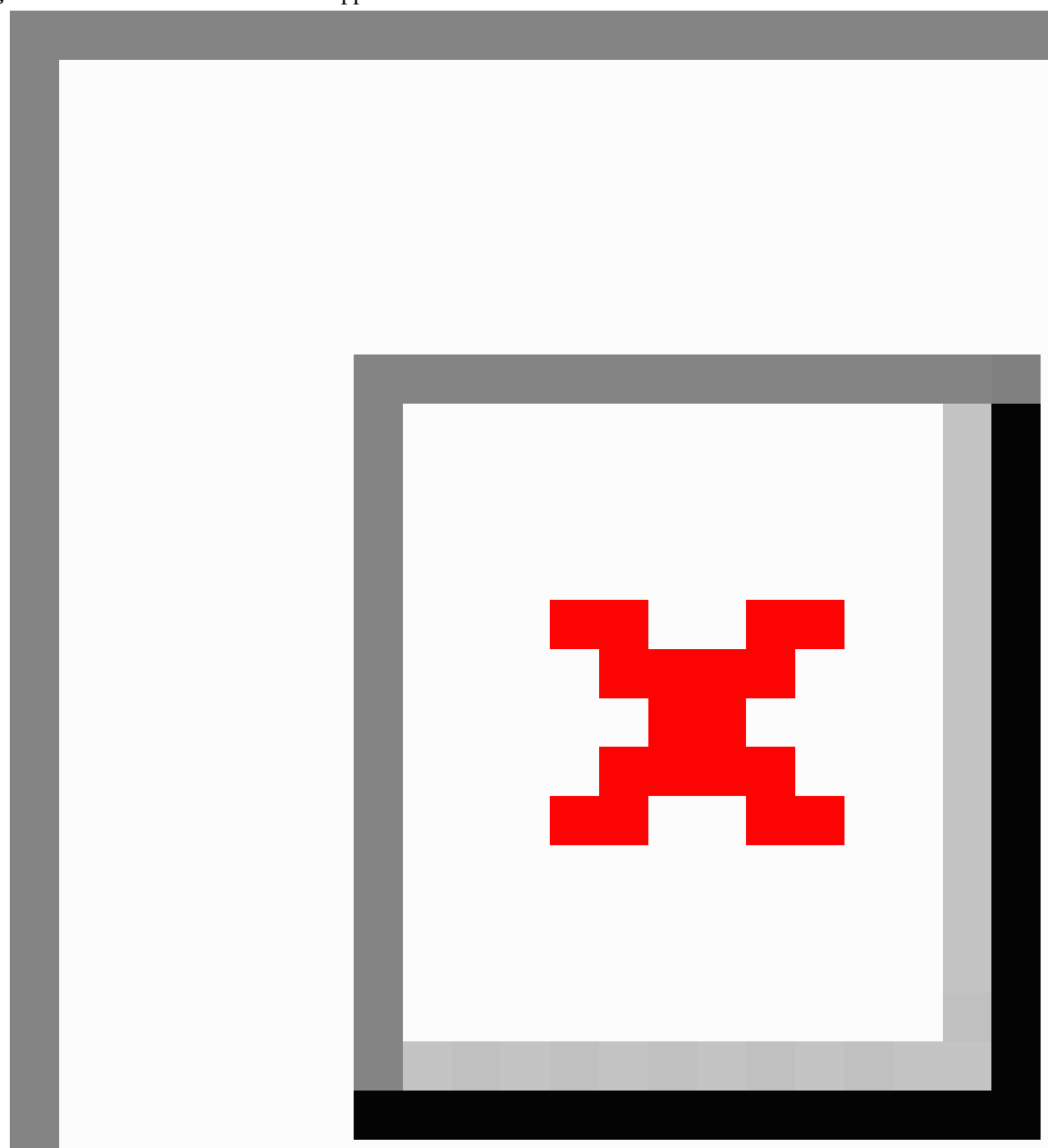

To help patients broaden their thoughts, the cognitive restructuring sessions provide four tools, each of which guides the patients to alternative thoughts through interaction with the characters. Each session is supposed to take 1 week: participants can proceed to the next session only after 1 week and only after they have completed one homework from that session. See Multimedia Appendix 3 for more details. One session requires approximately 20 minutes to complete.

The progress of each participant on Kokoro-app could be monitored at "Kokoro-Web." Participants and their treating psychiatrists could access their records using a unique identification number and password. Secure Sockets Layer certified security of the data exchanged through the Internet.
Every week, the central office sent an email to participants to congratulate them on their progress. The message was templated but individually modified based on the progress of participants and the comments they uploaded at the end of each session. Writing one such email took 3 to 10 minutes.

All study participants had access to the welcome session at week 0 . After randomization at week 1 , the intervention group received another password and continued with further sessions. The control group continued with the medication change only. Face-to-face CBT or interpersonal therapy were prohibited for either group. 


\section{Follow-Up Period After 9 Weeks}

After assessments at week 9, there were no further restrictions in medications or psychotherapies. The participants in the control arm received a password to begin the Kokoro-app; follow-up assessments took place at week 17.

\section{Outcomes}

The masked assessors interviewed the participants by telephone with PHQ-9 and FIBSER at weeks 0, 1, 5, 9, and 17.

The primary outcome was the PHQ-9 at week 9. It consists of the nine diagnostic criteria items of major depression [47]. Each item is rated between $0=$ "not at all" through $3=$ "nearly every day." The total score ranges between 0 and 27. The instrument has excellent reliability, validity, and responsiveness [48]. Remission was defined as scoring four or less on PHQ-9, and response as $50 \%$ or greater reduction from baseline.

Secondary outcomes included the FIBSER, which assesses the frequency, intensity, and burden of side effects, each on a 7-point scale, with a total score between 3 and 27, [49] and the BDI-II [43], a self-report measure of depression severity that asks about 21 symptoms of depression, each on a scale between 0 and 3 , with the total score between 0 and 63 .

The study participants and the psychiatrists in charge of medication change were aware of the treatment allocation. The outcome assessors conducting telephone interviews were unaware of allocation. The success of this masking was evaluated by calculating the Bang Index [50] of assessors' treatment guesses after each telephone assessment. The Bang Index is scaled to an interval of -1 to 1,1 being complete lack of blinding, 0 being consistent with perfect random guessing, and -1 indicating opposite guessing.

\section{Sample Size}

The study was powered to detect a moderate effect size of 0.5 in terms of standardized mean difference between the two treatments for the primary outcome at week 9 , with $80 \%$ power at two-sided alpha level of .05. Assuming that $30 \%$ would leave the study or be classified into the auxiliary group at week 1 , the required total sample size was 164 participants.

\section{Statistical Analysis}

All analyses were undertaken according to the intention-to-treat principle, including all the randomized participants for the total sample as well as for the per-protocol sample.

For each continuous outcome up to week 9, we used a linear mixed model including sites and patients as random effects and time (5 and 9 weeks), treatment, and time*treatment interaction, adjusting for its baseline score and the stratification variables, as fixed effects. The primary endpoint was the estimate of the least squares mean difference along with the $95 \%$ confidence interval $(95 \% \mathrm{CI})$ at week 9 . For the continuous outcome at week 17 follow-up, we used the similar linear mixed model but without the time*treatment interaction because this was a one-time comparison after all the participants received the smartphone CBT both in the intervention and the control groups. For the dichotomous outcomes, we used a generalized linear mixed model with the same random effects and the fixed effects. We chose odds ratios as the measure of effect. We used SAS version 9.4 (SAS Institute Inc, Cary, NC, USA). Multimedia Appendix 1 provides the statistical analysis plan.

\section{Blinded Interpretation of the Results}

The statistician (QZ), blinded to allocation, conducted the statistical analyses. The writing committee reviewed a statistical report in which the two treatment arms were designated A and $\mathrm{B}$, and developed interpretation of the results and associated conclusions under two different scenarios, one assuming A to be the smartphone CBT plus medication change arm and B to be the medication change alone arm, and another alternative scenario. The code was broken only after the writing committee signed off on the agreed-on interpretations (see Multimedia Appendix 4).

\section{Results}

\section{Participant Characteristics}

Figure 2 shows the flow of participants through the study. Between September 2, 2014, and July 1, 2016, 323 patients were assessed for eligibility; 166 patients provided informed consent and started medication change. Two withdrew consent before randomization at week 1 . Therefore, we recruited 164 patients, of whom 117 found no difficulty with the smartphone, were adherent to the protocol treatment, remained at least moderately symptomatic, and constituted the per-protocol sample; 60 were allocated to the smartphone CBT and 57 to medication change alone. Of the remaining 47 participants in the auxiliary group, 21 were allocated to the intervention group and 26 to control. Primary outcome data at 9 weeks were obtained from all but one randomized participant (163/164, $99.4 \%)$.

Table 1 shows that the baseline demographic and clinical characteristics of the treatment groups were well balanced. Typically, patients were in their thirties to forties, had three previous episodes, were in the current episode for nearly 2 years, and were severely to moderately depressed. 
Figure 2. Assessment, randomization, and follow-up of study participants. CBT: cognitive behavioral therapy; PHQ-9: Patient Health Questionnaire 9; TAU: treatment as usual.

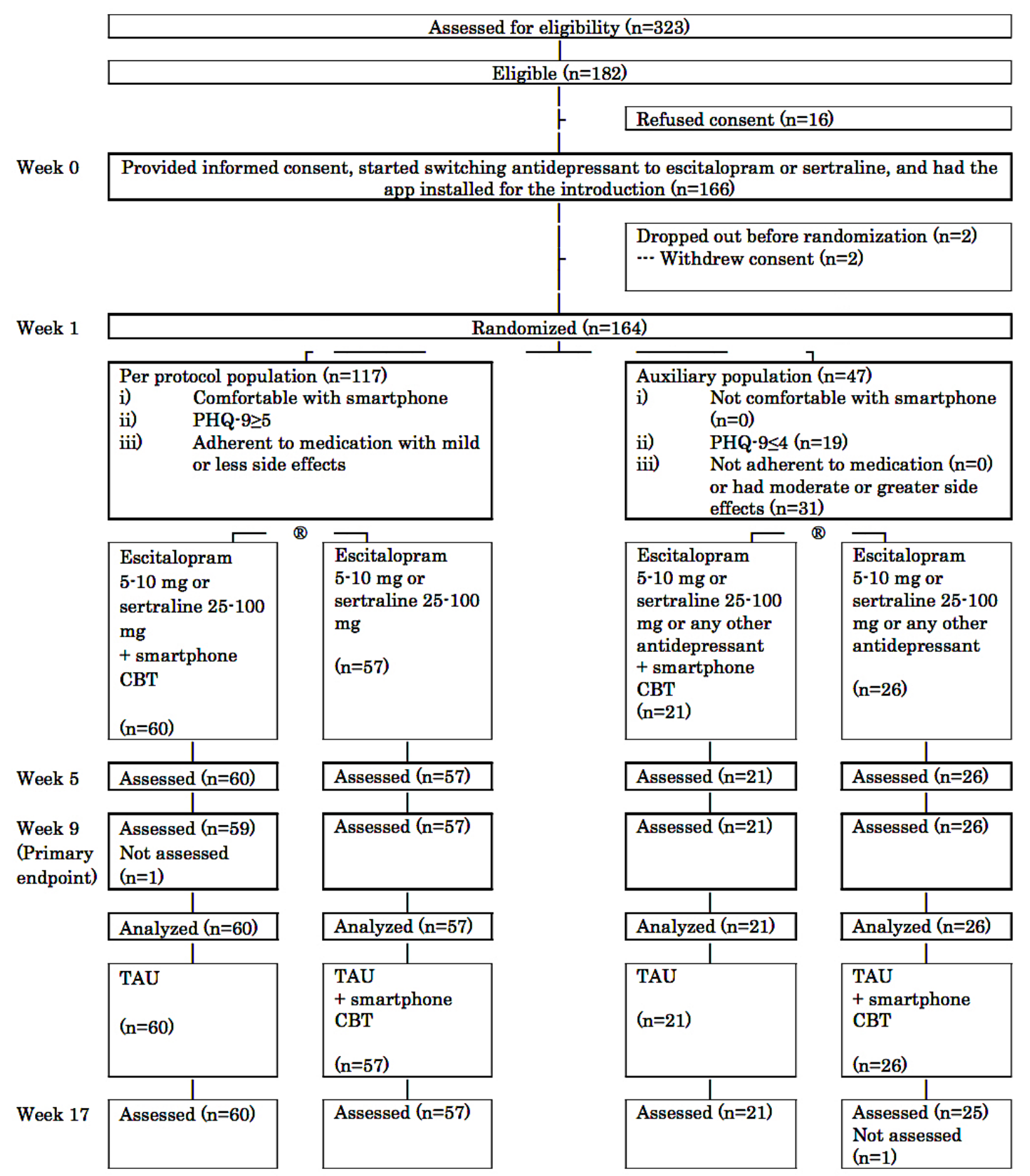


Table 1. Baseline characteristics of the intention-to-treat samples.

\begin{tabular}{|c|c|c|c|c|}
\hline \multirow[t]{2}{*}{ Characteristics } & \multicolumn{2}{|l|}{ Total sample $(\mathrm{N}=164)$} & \multicolumn{2}{|c|}{ Per-protocol sample $(\mathrm{n}=117)$} \\
\hline & $\begin{array}{l}\text { Smartphone } \mathrm{CBT}+ \\
\text { medication change } \\
(\mathrm{n}=81)\end{array}$ & $\begin{array}{l}\text { Medication change } \\
\text { alone }(\mathrm{n}=83)\end{array}$ & $\begin{array}{l}\text { Smartphone } \mathrm{CBT}+ \\
\text { medication change } \\
(\mathrm{n}=60)\end{array}$ & $\begin{array}{l}\text { Medication change } \\
\text { alone }(n=57)\end{array}$ \\
\hline \multicolumn{5}{|l|}{ Demographic characteristics } \\
\hline Age (years), mean (SD) & $40.2(8.8)$ & $41.6(8.9)$ & $40.1(9.0)$ & $41.2(8.6)$ \\
\hline Sex (female), n (\%) & $46(57)$ & $41(50)$ & $35(58)$ & $31(54)$ \\
\hline Education (years), mean (SD) & $14.6(2.5)$ & $14.9(2.7)$ & $14.8(2.5)$ & $15.1(2.9)$ \\
\hline \multicolumn{5}{|l|}{ Employment status, n (\%) } \\
\hline Employed full-time & $34(42)$ & $29(35)$ & $27(45)$ & $21(37)$ \\
\hline Employed part-mime & $7(9)$ & $5(6)$ & $5(8)$ & $5(9)$ \\
\hline On medical leave & $21(26)$ & $30(36)$ & $14(23)$ & $21(37)$ \\
\hline Housewife & $6(7)$ & $5(6)$ & $5(8)$ & $3(5)$ \\
\hline Student & 0 & $2(21)$ & 0 & $1(2)$ \\
\hline Retired & 0 & 0 & 0 & 0 \\
\hline Not employed & $13(16)$ & $12(15)$ & $9(15)$ & $6(11)$ \\
\hline \multicolumn{5}{|l|}{ Marital status, $n(\%)$} \\
\hline Single, never married & $34(42)$ & $31(38)$ & $24(40)$ & $22(39)$ \\
\hline Single, divorced, separated or widowed & $13(16)$ & $7(8)$ & $12(20)$ & $5(9)$ \\
\hline Married & $34(42)$ & $45(54)$ & $24(40)$ & $30(53)$ \\
\hline \multicolumn{5}{|l|}{ Baseline clinical characteristics, mean (SD) } \\
\hline Age of onset at first episode (years) & $31.8(10.8)$ & $34.6(10.0)$ & $32.2(11.0)$ & $34.0(10.2)$ \\
\hline Number of previous depressive episodes & $3.4(4.9)$ & $3.0(4.5)$ & $3.5(4.7)$ & $2.8(4.6)$ \\
\hline Length of current episode (months) & $24.2(46.3)$ & $23.0(46.5)$ & $27.0(52.6)$ & $22.3(42.5)$ \\
\hline \multicolumn{5}{|l|}{ PHQ-9 ${ }^{\text {a }}$} \\
\hline Week 0 & $13.5(5.5)$ & $12.9(5.3)$ & $14.0(5.2)$ & $13.8(5.1)$ \\
\hline Week 1 & $12.6(6.2)$ & $11.9(5.9)$ & $13.4(5.6)$ & $12.6(5.5)$ \\
\hline \multicolumn{5}{|l|}{ BDI-II ${ }^{a}$} \\
\hline Week 0 & $28.2(11.2)$ & $26.2(11.0)$ & $29.4(10.6)$ & $27.4(10.7)$ \\
\hline Week 1 & $26.2(11.7)$ & $24.7(12.2)$ & $28.1(11.0)$ & $26.2(11.6)$ \\
\hline \multicolumn{5}{|l|}{ FIBSER $^{a}$} \\
\hline Week 0 & $4.8(4.5)$ & $5.2(3.1)$ & $4.4(2.8)$ & $5.4(2.9)$ \\
\hline Week 1 & $6.4(4.5)$ & $6.8(4.4)$ & $5.0(3.0)$ & $5.2(2.5)$ \\
\hline
\end{tabular}

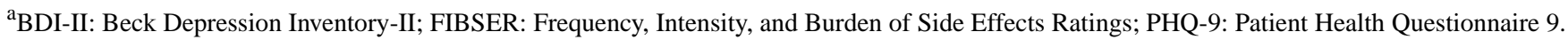

\section{Treatments Received}

In the total sample, 16 of 81 participants (20\%) stopped the protocol antidepressant treatment by escitalopram or sertraline in the intervention arm, as did 14 of $83(17 \%)$ in the medication only arm (Table 2). Some received augmentation drugs, such as antipsychotics or lithium, which were not allowed in the protocol for the antidepressant switch ( $n=15$ in the total sample), a few had deterioration or side effects and could not continue the protocol treatment $(n=5)$, and a few others got so well and did not want to continue with the protocol medication $(n=2)$. The medication dosages were comparable between the arms.

For the smartphone CBT, all but one participant (80/81, 99\%) completed at least half the sessions of the program, and 71 of $81(88 \%)$ completed at least six of eight sessions. It took the patients, on average, 10.8 (SD 4.2) days to complete one session. The patients filled in a mean 11.2 (SD 11.4) "mind maps" for self-monitoring, conducted a mean 14.4 (SD 17.1) behavioral activation "personal experiments," and generated a mean 6.1 (SD 6.0) alternative thoughts for cognitive restructuring (Table 2). 
Table 2. Treatment received in the intention-to-treat samples.

\begin{tabular}{|c|c|c|c|c|}
\hline \multirow[t]{2}{*}{ Therapy } & \multicolumn{2}{|l|}{ Total sample $(\mathrm{N}=164)$} & \multicolumn{2}{|c|}{ Per-protocol sample $(n=117)$} \\
\hline & $\begin{array}{l}\text { Smartphone CBT }+ \\
\text { medication change } \\
(n=81)\end{array}$ & $\begin{array}{l}\text { Medication change } \\
\text { alone }(n=83)\end{array}$ & $\begin{array}{l}\text { Smartphone CBT }+ \\
\text { medication change } \\
(n=60)\end{array}$ & $\begin{array}{l}\text { Medication change } \\
\text { alone }(n=57)\end{array}$ \\
\hline \multicolumn{5}{|l|}{ Pharmacotherapy } \\
\hline $\begin{array}{l}\text { Discontinuation of protocol antidepressant } \\
\text { treatment by escitalopram or sertraline by } \\
\text { week } 9, \mathrm{n}(\%)\end{array}$ & $16(20)$ & $14(17)$ & $10(17)$ & $8(14)$ \\
\hline \multicolumn{5}{|l|}{ Discontinuation reason, $\mathbf{n}$} \\
\hline Prescription of prohibited drugs & 6 & 9 & 4 & 5 \\
\hline Amelioration & 1 & 1 & - & 1 \\
\hline Deterioration & 1 & - & 1 & - \\
\hline Side effects & 3 & 1 & 1 & - \\
\hline Other & 5 & 3 & 4 & 2 \\
\hline $\begin{array}{l}\text { Discontinuation of any antidepressant ther- } \\
\text { apy by week } 9, \mathrm{n}(\%)\end{array}$ & $5(6)$ & $2(2)$ & $2(3)$ & $2(4)$ \\
\hline $\begin{array}{l}\text { Escitalopram dosage at week } 9 \text { (mg/day), } \\
\text { mean (SD) }\end{array}$ & $9.5(3.0), \mathrm{n}=48$ & $10.0(3.2), \mathrm{n}=49$ & $9.7(3.1), \mathrm{n}=38$ & $10.1(2.9), \mathrm{n}=34$ \\
\hline $\begin{array}{l}\text { Sertraline dosage at week } 9(\mathrm{mg} / \text { day }) \text {, mean } \\
\text { (SD) }\end{array}$ & $79.0(26.7), n=25$ & $83.6(23.4), n=29$ & $81.6(26.1), n=19$ & $83.0(24.9), n=22$ \\
\hline \multicolumn{5}{|l|}{ Smartphone CBT } \\
\hline Sessions completed, $n$ & & - & & - \\
\hline 0 & 1 & & - & \\
\hline 3 & 1 & & 1 & \\
\hline 4 & 4 & & 3 & \\
\hline 5 & 4 & & 2 & \\
\hline 6 & 11 & & 8 & \\
\hline 7 & 17 & & 13 & \\
\hline 8 & 43 & & 33 & \\
\hline Time per session (days), mean (SD) & $10.8(4.2)$ & - & $10.7(4.0)$ & - \\
\hline $\begin{array}{l}\text { Number of mind maps for self-monitoring, } \\
\text { mean (SD) }\end{array}$ & $11.2(11.4)$ & - & $10.8(10.3)$ & - \\
\hline $\begin{array}{l}\text { Number of behavioral activation tasks, } \\
\text { mean (SD) }\end{array}$ & $14.4(17.1)$ & - & $14.6(17.8)$ & - \\
\hline Number of alternative thoughts, mean (SD) & $6.1(6.0)$ & - & $6.5(6.0)$ & - \\
\hline
\end{tabular}


Table 3. Outcomes at weeks 9 and 17 for the total sample $(\mathrm{N}=164)$.

\begin{tabular}{|c|c|c|c|c|}
\hline Outcomes $^{\mathrm{a}}$ & $\begin{array}{l}\text { Smartphone CBT }+ \text { medication change } \\
(\mathrm{n}=81), \text { mean } / \%(95 \% \mathrm{CI})\end{array}$ & $\begin{array}{l}\text { Medication change alone } \\
(\mathrm{n}=83), \text { mean } / \%(95 \% \mathrm{CI})\end{array}$ & $\begin{array}{l}\text { Adjusted difference/OR } \\
(95 \% \mathrm{CI})^{\mathrm{b}}\end{array}$ & $P$ value \\
\hline \multicolumn{5}{|c|}{ End of randomized trial (week 9) } \\
\hline PHQ-9 & $7.94(6.98,8.89)$ & $10.41(9.45,11.33)$ & $-2.48(-3.72,-1.23)$ & $<.001$ \\
\hline Remission & $30.5 \%(19.7 \%, 43.9 \%)$ & $17.8 \%(10.3 \%, 29.0 \%)$ & $2.02(0.93,4.42)$ & .08 \\
\hline Response & $42.3 \%(29.4 \%, 56.4 \%)$ & $21.2 \%(12.7 \%, 33.2 \%)$ & $2.73(1.35,5.53)$ & .005 \\
\hline BDI-II & $19.3(17.0,21.5)$ & $23.3(21.6,25.5)$ & $-4.1(-6.6,-1.5)$ & .002 \\
\hline FIBSER & $4.38(3.72,5.03)$ & $5.14(4.52,5.76)$ & $-0.76(-1.58,0.05)$ & .07 \\
\hline \multicolumn{5}{|c|}{ Follow-up (week 17) } \\
\hline PHQ-9 & $7.95(6.73,9.17)$ & $8.76(7.58,9.95)$ & $-0.81(-2.24,0.62)$ & .26 \\
\hline BDI-II & $17.2(14.4,20.0)$ & $19.1(16.4,21.8)$ & $-1.9(-4.9,1.2)$ & .22 \\
\hline FIBSER & $4.62(3.83,5.42)$ & $5.10(4.32,5.89)$ & $-0.48(-1.34,0.37)$ & .27 \\
\hline
\end{tabular}

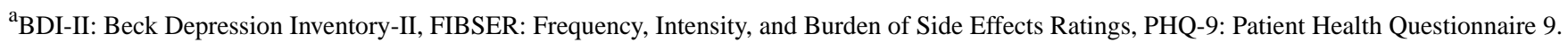

${ }^{b}$ For each continuous outcome up to week 9 , we used a linear mixed model including sites and patients as random effects and time (5 and 9 weeks), treatment, and time*treatment interaction, adjusting for its baseline score and the stratification variables, as fixed effects. For the continuous outcome at week 17 follow-up, we used the similar linear mixed model but without time*treatment interaction. For the dichotomous outcomes, the generalized linear mixed model was used along with the same random effects and the fixed effects. The summary effect measures are adjusted score differences for PHQ-9, BDI-II, and FIBSER, and are odds ratios for remission and response.

\section{Outcomes for the Total Sample}

In the intention-to-treat analysis of the total sample, patients who were allocated the CBT app $(n=81)$ scored 2.48 points (95\% CI 1.23-3.72, $P<.001$; standardized mean difference [SMD] 0.40) lower on PHQ-9 than those who were not $(n=83)$ at week 9 (Table 3, Figure 3). The former group also scored 4.1 points (95\% CI 1.5-6.6, $P=.002)$ lower on BDI-II. Significantly more participants showed response (OR 2.73, 95\% CI 1.35-5.53, $P=.005)$. However, the increase in remission did not reach statistical significance (OR 2.02, 95\% CI 0.93-4.42, $P=.08$ ).

With regard to the harm outcomes, patients using the smartphone CBT reported somewhat less overall burden of side effects, but the difference was not statistically significant (FIBSER mean difference $=-0.76,95 \% \mathrm{CI}-1.58$ to $0.05, P=.07)$. There was one report of suicidality (self-injurious behavior without suicidal intent) in the combined treatment arm and one report of a serious adverse event in the control arm (brief hospital admission for examination of preexisting spinal canal stenosis).

\section{Outcomes for the Per-Protocol Sample}

In the per-protocol sample, who were comfortable with the smartphone app, were still symptomatic, and were adherent to medication with mild or less side effects after the run-in, the patients who received smartphone CBT in addition to medication change $(n=60)$ scored mean 1.72 (95\% CI 0.25-3.18, $P=.02$; SMD 0.28) points lower on PHQ-9 than those undergoing medication change alone $(n=57)$ at week 9 . The combined treatment arm was superior to the control arm in terms of BDI-II (difference $=-3.2,95 \% \mathrm{CI}-6.3$ to $0.0, P=.05$, not statistically significant), but not in terms of remission (OR 1.99, 95\% CI 0.74-5.38, $P=.17$ ) or response (OR 2.11, 95\% CI 0.92-4.85, $P=.08)$.

In terms of the harm outcome, the combination treatment arm reported significantly less overall burden of side effects (FIBSER mean difference $=-0.75,95 \% \mathrm{CI}-1.47$ to $-0.03, P=.04$ ) (Table 4, Figure 3).

\section{Follow-Up at Week 17}

The participants who had access to the smartphone app in the first 9 weeks maintained their gains for a further 8 weeks. At week 17, when the participants in the control arm also had access to the smartphone app, the results were comparable between the two groups in terms of PHQ-9, BDI-II, and FIBSER, both for the total sample and for the per-protocol sample (Tables 3 and 4).

\section{Masking}

The Bang Index of the treatment guesses by the masked assessors at weeks 5,9 , and 17 was 0.10 (95\% CI-0.13 to 0.33$)$, 0.29 (95\% CI $0.06-0.51)$, and 0.30 (95\% CI 0.08-0.52) for the intervention arm and -0.21 (95\% CI -0.45 to 0.03$),-0.18$ (95\% CI -0.41 to 0.06 ), and -0.30 (95\% CI -0.53 to -0.07 ) for the control arm, respectively. The observed patterns indicate that the raters guessed the treatment allocation haphazardly or guessed it to be the smartphone CBT arm more often regardless of the actual allocation, resulting in ideally unbiased assessment of outcomes in the trial [51]. 
Figure 3. Trajectory of the Patient Health Questionnaire-9 (PHQ-9), Beck Depression Inventory-II (BDI-II), and Frequency, Intensity, and Burden of Side Effects Ratings (FIBSER) scores for the intervention (blue line) and control (red line) groups in the total (N=164) and per-protocol ( $\mathrm{n}=117)$ samples. Error bars show standard errors for model-based least squares means.

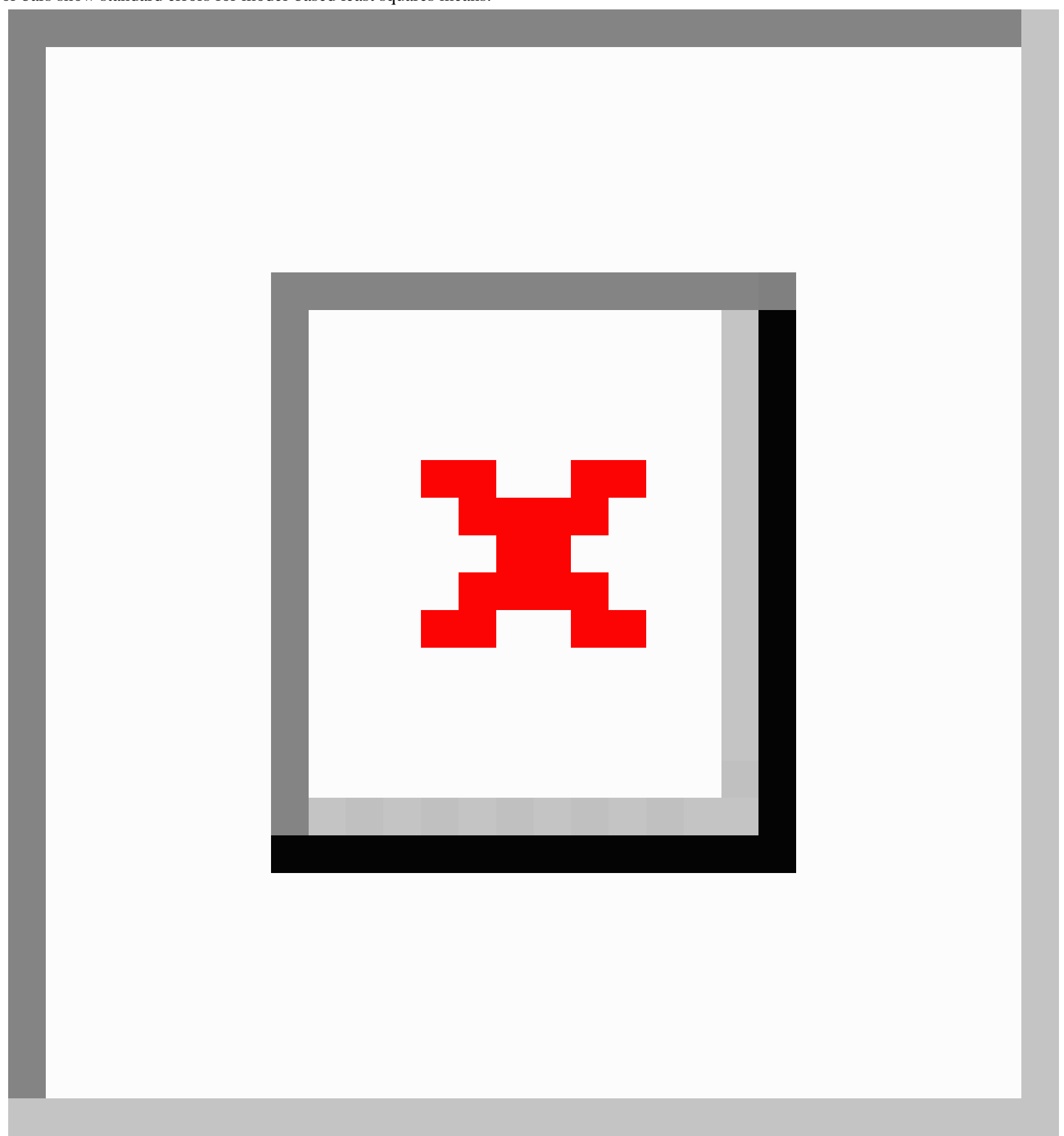


Table 4. Outcomes at weeks 9 and 17 for the per-protocol sample $(n=117)$.

\begin{tabular}{|c|c|c|c|c|}
\hline Outcomes $^{\mathrm{a}}$ & $\begin{array}{l}\text { Smartphone CBT + medication change } \\
(\mathrm{n}=60), \text { mean } / \%(95 \% \mathrm{CI})\end{array}$ & $\begin{array}{l}\text { Medication change alone } \\
(\mathrm{n}=57), \text { mean } / \%(95 \% \mathrm{CI})\end{array}$ & $\begin{array}{l}\text { Adjusted difference/OR } \\
(95 \% \mathrm{CI})^{\mathrm{b}}\end{array}$ & $P$ value \\
\hline \multicolumn{5}{|c|}{ End of randomized trial (week 9) } \\
\hline PHQ-9 & $8.92(7.81,10.03)$ & $10.64(9.52,11.76)$ & $-1.72(-3.18,-0.25)^{\mathrm{c}}$ & .02 \\
\hline Remission & $18.2 \%(8.5 \%, 34.8 \%)$ & $10.0 \%(4.0 \%, 23.2 \%)$ & $1.99(0.74,5.38)$ & .17 \\
\hline Response & $31.6 \%(18.7 \%, 48.3 \%)$ & $18.0 \%(9.2 \%, 32.3 \%)$ & $2.11(0.92,4.85)$ & .08 \\
\hline BDI-II & $21.0(18.6,23.5)$ & $24.2(21.7,26.8)$ & $-3.2(-6.3,0.0)$ & .05 \\
\hline FIBSER & $4.11(3.44,4.78)$ & $4.86(4.18,5.53)$ & $-0.75(-1.47,-0.03)$ & .04 \\
\hline \multicolumn{5}{|c|}{ Follow-up (week 17) } \\
\hline PHQ-9 & $8.92(7.40,10.44)$ & $8.85(7.31,10.39)$ & $0.07(-1.68,1.82)$ & .94 \\
\hline BDI-II & $19.4(15.9,22.9)$ & $20.0(16.5,23.6)$ & $-0.6(-4.4,3.1)$ & .75 \\
\hline FIBSER & $4.14(3.40,4.88)$ & $4.46(3.71,5.21)$ & $-0.32(-1.28,0.63)$ & .50 \\
\hline
\end{tabular}

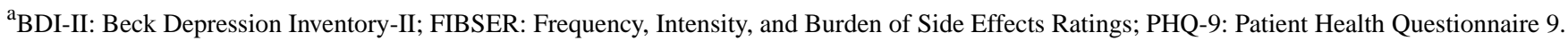

${ }^{\mathrm{b}}$ For each continuous outcome up to week 9 , we used a linear mixed model including sites and patients as random effects and time (5 and 9 weeks), treatment, and time*treatment interaction, adjusting for its baseline score and the stratification variables, as fixed effects. For the continuous outcome at week 17 follow-up, we used the similar linear mixed model but without time*treatment interaction. For the dichotomous outcomes, the generalized linear mixed model was used along with the same random effects and the fixed effects. The summary effect measures are adjusted score differences for PHQ-9, BDI-II, and FIBSER, and are odds ratios for remission and response.

${ }^{\mathrm{c}}$ Primary endpoint per protocol.

\section{Discussion}

In patients with major depression who had not responded to one or more antidepressants, adding smartphone CBT to medication change was more effective than treatment by medication change alone. The smartphone CBT also decreased the overall side effect burden of the pharmacotherapy.

The magnitude of benefit of the adjunctive mobile CBT was approximately 2 points on the PHQ- 9 and 3 to 4 points on the BDI-II. Using the observed standard deviation at week 9, these differences translate into standardized mean differences of 0.28 to 0.40 and are comparable to that of 0.31 for antidepressants over placebo reported in a comprehensive systematic review of phase II or III RCTs [52]. The remission and response rates almost doubled, corresponding with numbers needed to treat between 5 and 12 .

Although several smartphone CBT apps have been experimented among general population participants with elevated self-reported symptoms [27-33], Kokoro-app is the first smartphone CBT app to prove to be effective in a RCT in comparison with an alternative treatment for patients suffering from clinically diagnosed major depression. Advantages of smartphone CBT include high accessibility, efficiency, and affordability. Further, it is less susceptible to quality control problems that may plague face-to-face therapies [53].

Unexpectedly, Kokoro-app also reduced the global burden of side effects due to pharmacotherapy. We speculate that the smartphone CBT, through which the patient actively searches for ways to overcome their depression, may increase their sense of self-control and decrease the subjective burden of side effects in comparison with standard pharmacotherapy.
All benefits were larger in the total sample than in the per-protocol comparison, where we had anticipated a larger effect at the protocol stage. Participants were not included in the per-protocol sample mainly because they suffered from side effects from the medication change (31 of 47, see Figure 1). The CBT app may be particularly beneficial to those patients who experience significant side effects with medication.

Strengths of this study include the concealed randomization, the successful masking of the outcome assessors, the close to $100 \%$ follow-up, the stratified randomization that allowed assessment of intervention impact in two key samples, and corroboration of the secondary outcomes including patients' self-reports. We followed the participants for 8 weeks after the end of the randomized comparison: the participants in the active intervention arm maintained the benefits, and the participants in the control arm who had access to Kokoro-app improved. Finally, the uptake of the CBT sessions via smartphone was satisfactory, with close to $90 \%$ of the participants finishing at least six of eight program sessions and actively engaging in homework tasks.

This study is not without limitations. First, it is possible that participants in the control condition, who were not allowed access to CBT sessions at the beginning but only after the waiting period, may have suffered "disappointment effect" through the intervention period. We tried to mitigate this limitation that may be common to many waiting list-controlled trials by not making our control condition a simple waiting list control in which the participants are not allowed to change their treatment [54], but an active medication change, which is one of the recommended treatment options for antidepressant-refractory patients. Nonetheless, the possibility of some contribution of disappointment effect among the control group cannot be negated. Secondly, it was impossible to mask 
the participants and the clinicians administering drug treatment to the treatment allocation. However, we employed the remote telephone assessors to conduct assessments of the primary outcome, which resulted in successful masking. Lastly, it must be pointed out that in this refractory population, although the smartphone CBT in conjunction with medication switch substantively decreased depression severity, approximately $70 \%$ to $80 \%$ were still not remitted after 2 months of the combined treatment; additional face-to-face standard CBT may be helpful for these remaining patients.

These findings have demonstrated the effectiveness of smartphone CBT as an adjunctive intervention for antidepressant-resistant major depression. Given the merits of the mobile mental health intervention, including accessibility, affordability, quality control, and effectiveness, patients and clinicians may wish to use smartphone CBT as an adjunctive intervention when their depression does not respond adequately to antidepressant treatment alone. Further research of its effectiveness in wider clinical contexts, including its use as a stand-alone treatment in major depression and its role in relapse prevention, and in public health contexts such as its use for subthreshold depressive states or its utility in low- and middle-income countries is warranted. Elucidation of the effective components of the smartphone CBT package and their appropriate dosages and their integration within the existing health care systems also constitute areas needing further research.

\section{Acknowledgments}

The study was funded by the Ministry of Health, Labor and Welfare, Japan (H-22-Seishin-Ippan-008) from April 2013 through March 2014, and thereafter by the Japan Foundation for Neuroscience and Mental Health (JFNMH). The JFNMH received donations from Asahi Kasei, Eli Lilly, GSK, Janssen, MSD, Meiji Seika, Mochida, Otsuka, Pfizer, Shionogi, Taisho, and Mitsubishi-Tanabe. The funders had no role in the design and conduct of the study; collection, management, analysis, and interpretation of the data; preparation, review, or approval of the manuscript; and decision to submit the manuscript for publication.

\section{Authors' Contributions}

TAF and QZ had full access to all the data in the study and take responsibility for the integrity of the data and the accuracy of the data analysis. Study concept and design: TAF, MH, and NY. Acquisition, analysis, or interpretation of data: AM, TK, TAF, MH, HI, TH, BC, TF, NY, QZ, and NK. Drafting of the manuscript: TAF. Critical revision of the manuscript for important intellectual content: AM, TK, TAF, MH, HI, TH, BC, TF, NY, QZ, and NK. Statistical analysis: QZ and TAF. Obtained funding: TAF. Administrative, technical, or material support: HI, NY, and NK. Study supervision: TAF and MH.

\section{Conflicts of Interest}

AM has received lecture fees from Mochida, Eli Lilly, and Meiji. TK has received lecture fees from Eli Lilly and Mitsubishi-Tanabe, and has contracted research with GlaxoSmithKline, MSD, and Mitsubishi-Tanabe. TAF has received lecture fees from Eli Lilly, Janssen, Meiji, MSD, Otsuka, Pfizer, and Mitsubishi-Tanabe, and consultancy fees from Takeda Science Foundation. He has received research support from Mochida and Mitsubishi-Tanabe. TH has received lecture fees from Otsuka and MSD. BC has received lecture fees from Eli Lilly, Meiji, and Mitsubishi-Tanabe. TF has received lecture fees from Eli Lilly, Meiji, MSD, Otsuka, Pfizer, and Mitsubishi-Tanabe. TAF and MH developed the Kokoro-app. All the other authors declare that they have no competing interests.

\section{Multimedia Appendix 1}

Original study protocol (August 2014) and statistical analysis plan (July 2016).

[PDF File (Adobe PDF File), 275KB-Multimedia Appendix 1]

\section{Multimedia Appendix 2}

FLATT investigators and committee members.

[PDF File (Adobe PDF File), 77KB-Multimedia Appendix 2]

\section{Multimedia Appendix 3}

Contents of Kokoro-app.

[PDF File (Adobe PDF File), 824KB-Multimedia Appendix 3]

\section{Multimedia Appendix 4}

Blinded data analyses statement of interpretation. 


\section{Multimedia Appendix 5}

CONSORT - EHEALTH checklist (V.1.6.1).

[PDF File (Adobe PDF File), 8MB-Multimedia Appendix 5]

\section{References}

1. Bromet E, Andrade LH, Hwang I, Sampson NA, Alonso J, de Girolamo G, et al. Cross-national epidemiology of DSM-IV major depressive episode. BMC Med 2011 Jul 26;9:90 [FREE Full text] [doi: 10.1186/1741-7015-9-90] [Medline: 21791035]

2. Ferrari AJ, Charlson FJ, Norman RE, Patten SB, Freedman G, Murray CJL, et al. Burden of depressive disorders by country, sex, age, and year: findings from the global burden of disease study 2010. PLoS Med 2013 Nov;10(11):e1001547 [FREE Full text] [doi: 10.1371/journal.pmed.1001547] [Medline: 24223526]

3. Bloom D, Cafiero E, Jane-Llopis E, Abrahams-Gessel S, Bloom L, Fathima S. The Global Economic Burden of Noncommunicable Diseases. Geneva: World Economic Forum; 2011.

4. Mathers CD, Loncar D. Projections of global mortality and burden of disease from 2002 to 2030. PLoS Med 2006 Nov;3(11):e442 [FREE Full text] [doi: 10.1371/journal.pmed.0030442] [Medline: 17132052]

5. Demyttenaere K, Bruffaerts R, Posada-Villa J, Gasquet I, Kovess V, Lepine JP, et al. Prevalence, severity, and unmet need for treatment of mental disorders in the World Health Organization World Mental Health Surveys. JAMA 2004 Jun 2;291(21):2581-2590. [doi: 10.1001/jama.291.21.2581] [Medline: 15173149]

6. Collins PY, Patel V, Joestl SS, March D, Insel TR, Daar AS, Scientific Advisory Board and the Executive Committee of the Grand Challenges on Global Mental Health, et al. Grand challenges in global mental health. Nature 2011 Jul 06;475(7354):27-30 [FREE Full text] [doi: 10.1038/475027a] [Medline: 21734685]

7. Marcus SC, Olfson M. National trends in the treatment for depression from 1998 to 2007. Arch Gen Psychiatry 2010 Dec;67(12):1265-1273. [doi: 10.1001/archgenpsychiatry.2010.151] [Medline: 21135326]

8. Trivedi MH, Rush AJ, Wisniewski SR, Nierenberg AA, Warden D, Ritz L, STAR*D Study Team. Evaluation of outcomes with citalopram for depression using measurement-based care in STAR*D: implications for clinical practice. Am J Psychiatry 2006 Jan;163(1):28-40. [doi: 10.1176/appi.ajp.163.1.28] [Medline: 16390886]

9. American Psychiatric Association Work Group on Major Depressive Disorder. Practice Guideline for the Treatment of Patients with Major Depressive Disorder. Third Edition. Arlington, VA: American Psychiatric Association; 2010.

10. NICE. Depression: The Treatment and Management of Depression in Adults (partial update of NICE clinical guideline 23). London: National Institute for Clinical Excellence; 2009.

11. Furukawa T, Weitz E, Tanaka S, Hollon S, Hofmann S, Andersson G, et al. Initial severity of depression and efficacy of cognitive-behavioural therapy: individual-participant data meta-analysis of pill-placebo-controlled trials. Br J Psychiatry 2017 Mar;210(3):190-196. [doi: 10.1192/bjp.bp.116.187773] [Medline: 28104735]

12. Cuijpers P, Dekker J, Hollon SD, Andersson G. Adding psychotherapy to pharmacotherapy in the treatment of depressive disorders in adults: a meta-analysis. J Clin Psychiatry 2009 Sep;70(9):1219-1229. [doi: 10.4088/JCP.09r05021] [Medline: 19818243]

13. Cuijpers P, van Straten A, Warmerdam L, Andersson G. Psychotherapy versus the combination of psychotherapy and pharmacotherapy in the treatment of depression: a meta-analysis. Depress Anxiety 2009;26(3):279-288. [doi: 10.1002/da.20519] [Medline: 19031487]

14. Wang PS, Aguilar-Gaxiola S, Alonso J, Angermeyer MC, Borges G, Bromet EJ, et al. Use of mental health services for anxiety, mood, and substance disorders in 17 countries in the WHO world mental health surveys. Lancet 2007 Sep 8;370(9590):841-850 [FREE Full text] [doi: 10.1016/S0140-6736(07)61414-7] [Medline: 17826169]

15. Olfson M, Marcus SC. National trends in outpatient psychotherapy. Am J Psychiatry 2010 Dec;167(12):1456-1463. [doi: 10.1176/appi.ajp.2010.10040570] [Medline: 20686187]

16. Cuijpers P, Donker T, van Straten A, Li J, Andersson G. Is guided self-help as effective as face-to-face psychotherapy for depression and anxiety disorders? A systematic review and meta-analysis of comparative outcome studies. Psychol Med 2010 Dec;40(12):1943-1957. [doi: 10.1017/S0033291710000772] [Medline: 20406528]

17. Karyotaki E, Riper H, Twisk J, Hoogendoorn A, Kleiboer A, Mira A, et al. Efficacy of self-guided Internet-based cognitive behavioral therapy in the treatment of depressive symptoms: a meta-analysis of individual participant data. JAMA Psychiatry 2017 Apr 01;74(4):351-359. [doi: 10.1001/jamapsychiatry.2017.0044] [Medline: 28241179]

18. Gilbody S, Littlewood E, Hewitt C, Brierley G, Tharmanathan P, Araya R, et al. Computerised cognitive behaviour therapy (cCBT) as treatment for depression in primary care (REEACT trial): large scale pragmatic randomised controlled trial. BMJ 2015;351:h5627 [FREE Full text] [Medline: 26559241]

19. IMS Institute for Healthcare Informatics. 2015. Patient options expand as mobile healthcare apps address wellness and chronic disease treatment needs URL: http://www.imshealth.com/en/thought-leadership/quintilesims-institute/reports/ patient-options-expand-as-mobile-healthcare-apps-address-wellness-and-chronic-disease-treatment-needs [accessed 2017-07-29] [WebCite Cache ID 6sJIcCKHV]

20. Rathbone AL, Prescott J. The use of mobile apps and SMS messaging as physical and mental health interventions: systematic review. J Med Internet Res 2017 Aug 24;19(8):e295 [FREE Full text] [doi: 10.2196/jmir.7740] [Medline: 28838887] 
21. Wahle F, Bollhalder L, Kowatsch T, Fleisch E. Toward the design of evidence-based mental health information systems for people with depression: a systematic literature review and meta-analysis. J Med Internet Res 2017 May 31;19(5):e191 [FREE Full text] [doi: 10.2196/jmir.7381] [Medline: 28566267]

22. Anthes E. Mental health: there's an app for that. Nature 2016 Apr 7;532(7597):20-23. [doi: 10.1038/532020a] [Medline: 27078548]

23. Torous J, Roberts LW. Needed innovation in digital health and smartphone applications for mental health: transparency and trust. JAMA Psychiatry 2017 May 01;74(5):437-438. [doi: 10.1001/jamapsychiatry.2017.0262] [Medline: 28384700]

24. Kauer SD, Reid SC, Crooke AH, Khor A, Hearps SJ, Jorm AF, et al. Self-monitoring using mobile phones in the early stages of adolescent depression: randomized controlled trial. J Med Internet Res 2012;14(3):e67 [FREE Full text] [doi: 10.2196/jmir.1858] [Medline: 22732135]

25. Proudfoot J, Clarke J, Birch M, Whitton AE, Parker G, Manicavasagar V, et al. Impact of a mobile phone and web program on symptom and functional outcomes for people with mild-to-moderate depression, anxiety and stress: a randomised controlled trial. BMC Psychiatry 2013;13:312 [FREE Full text] [doi: 10.1186/1471-244X-13-312] [Medline: 24237617]

26. Reid SC, Kauer SD, Hearps SJ, Crooke AH, Khor AS, Sanci LA, et al. A mobile phone application for the assessment and management of youth mental health problems in primary care: a randomised controlled trial. BMC Fam Pract 2011;12:131 [FREE Full text] [doi: 10.1186/1471-2296-12-131] [Medline: 22123031]

27. Bush NE, Smolenski DJ, Denneson LM, Williams HB, Thomas EK, Dobscha SK. A virtual hope box: randomized controlled trial of a smartphone app for emotional regulation and coping with distress. Psychiatr Serv 2017 Apr 01;68(4):330-336. [doi: 10.1176/appi.ps.201600283] [Medline: 27842473]

28. Arean PA, Hallgren KA, Jordan JT, Gazzaley A, Atkins DC, Heagerty PJ, et al. The use and effectiveness of mobile apps for depression: results from a fully remote clinical trial. J Med Internet Res 2016 Dec 20;18(12):e330 [FREE Full text] [doi: 10.2196/jmir.6482] [Medline: 27998876]

29. Roepke AM, Jaffee SR, Riffle OM, McGonigal J, Broome R, Maxwell B. Randomized controlled trial of SuperBetter, a smartphone-based/Internet-based self-help tool to reduce depressive symptoms. Games Health J 2015 Jun;4(3):235-246. [doi: 10.1089/g4h.2014.0046] [Medline: 26182069]

30. Birney AJ, Gunn R, Russell JK, Ary DV. MoodHacker mobile web app with email for adults to self-manage mild-to-moderate depression: randomized controlled trial. JMIR Mhealth Uhealth 2016 Jan 26;4(1):e8 [FREE Full text] [doi: 10.2196/mhealth.4231] [Medline: 26813737]

31. Fitzpatrick KK, Darcy A, Vierhile M. Delivering Cognitive Behavior Therapy to Young Adults With Symptoms of Depression and Anxiety Using a Fully Automated Conversational Agent (Woebot): A Randomized Controlled Trial. JMIR Ment Health 2017 Jun 06;4(2):e19 [FREE Full text] [doi: 10.2196/mental.7785] [Medline: 28588005]

32. Buntrock C, Berking M, Smit F, Lehr D, Nobis S, Riper H, et al. Preventing depression in adults with subthreshold depression: health-economic evaluation alongside a pragmatic randomized controlled trial of a web-based intervention. J Med Internet Res 2017 Jan 04;19(1):e5 [FREE Full text] [doi: 10.2196/jmir.6587] [Medline: 28052841]

33. Carolan S, Harris PR, Cavanagh K. Improving employee well-being and effectiveness: systematic review and meta-analysis of web-based psychological interventions delivered in the workplace. J Med Internet Res 2017 Jul 26;19(7):e271 [FREE Full text] [doi: 10.2196/jmir.7583] [Medline: 28747293]

34. Watts S, Mackenzie A, Thomas C, Griskaitis A, Mewton L, Williams A, et al. CBT for depression: a pilot RCT comparing mobile phone vs computer. BMC Psychiatry 2013;13:49 [FREE Full text] [doi: 10.1186/1471-244X-13-49] [Medline: 23391304]

35. Ly KH, Topooco N, Cederlund H, Wallin A, Bergström J, Molander O, et al. Smartphone-supported versus full behavioural activation for depression: a randomised controlled trial. PLoS One 2015;10(5):e0126559 [FREE Full text] [doi: 10.1371/journal.pone.0126559] [Medline: 26010890]

36. Ly KH, Trüschel A, Jarl L, Magnusson S, Windahl T, Johansson R, et al. Behavioural activation versus mindfulness-based guided self-help treatment administered through a smartphone application: a randomised controlled trial. BMJ Open 2014;4(1):e003440 [FREE Full text] [doi: 10.1136/bmjopen-2013-003440] [Medline: 24413342]

37. Furukawa TA, Horikoshi M, Kawakami N, Kadota M, Sasaki M, Sekiya Y, GENKI Project. Telephone cognitive-behavioral therapy for subthreshold depression and presenteeism in workplace: a randomized controlled trial. PLoS One 2012;7(4):e35330 [FREE Full text] [doi: 10.1371/journal.pone.0035330] [Medline: 22532849]

38. Takagaki K, Okamoto Y, Jinnin R, Mori A, Nishiyama Y, Yamamura T, et al. Behavioral activation for late adolescents with subthreshold depression: a randomized controlled trial. Eur Child Adolesc Psychiatry 2016 Nov;25(11):1171-1182. [doi: 10.1007/s00787-016-0842-5] [Medline: 27003390]

39. Simon GE, Ludman EJ, Tutty S, Operskalski B, Von Korff M. Telephone psychotherapy and telephone care management for primary care patients starting antidepressant treatment: a randomized controlled trial. JAMA 2004 Aug 25;292(8):935-942. [doi: 10.1001/jama.292.8.935] [Medline: 15328325$]$

40. Watanabe N, Horikoshi M, Yamada M, Shimodera S, Akechi T, Miki K, Steering Committee of the Fun to Learn to ActThink through Technology Project. Adding smartphone-based cognitive-behavior therapy to pharmacotherapy for major depression (FLATT project): study protocol for a randomized controlled trial. Trials 2015 Jul 07;16:293 [FREE Full text] [doi: 10.1186/s13063-015-0805-z] [Medline: 26149441] 
41. American Psychiatric Association. Diagnostic and Statistical Manual of Mental Disorders. Fifth Edition. Arlington, VA: American Psychiatric Association; 2013.

42. Spitzer RL, Williams JB, Kroenke K, Linzer M, deGruy FV, Hahn SR, et al. Utility of a new procedure for diagnosing mental disorders in primary care. The PRIME-MD 1000 study. JAMA 1994 Dec 14;272(22):1749-1756. [Medline: 7966923]

43. Beck A, Steer R, Brown G. BDI-II: Beck Depression Inventory. Second Edition. San Antonio, TX: The Psychological Corporation; 1996.

44. Thase ME, Rush AJ. When at first you don't succeed: sequential strategies for antidepressant nonresponders. J Clin Psychiatry 1997;58 Suppl 13:23-29 [FREE Full text] [Medline: 9402916]

45. Kroenke K, Spitzer RL, Williams JB. The PHQ-9: validity of a brief depression severity measure. J Gen Intern Med 2001 Sep;16(9):606-613 [FREE Full text] [Medline: 11556941]

46. Cipriani A, Furukawa TA, Salanti G, Geddes JR, Higgins JP, Churchill R, et al. Comparative efficacy and acceptability of 12 new-generation antidepressants: a multiple-treatments meta-analysis. Lancet 2009 Feb 28;373(9665):746-758. [doi: 10.1016/S0140-6736(09)60046-5] [Medline: 19185342]

47. Spitzer RL, Kroenke K, Williams JB. Validation and utility of a self-report version of PRIME-MD: the PHQ primary care study. Primary Care Evaluation of Mental Disorders. Patient Health Questionnaire. JAMA 1999 Nov 10;282(18):1737-1744. [Medline: 10568646 ]

48. Furukawa TA. Assessment of mood: guides for clinicians. J Psychosom Res 2010 Jun;68(6):581-589. [doi: 10.1016/j.jpsychores.2009.05.003] [Medline: 20488276]

49. Rush AJ, Trivedi MH, Wisniewski SR, Stewart JW, Nierenberg AA, Thase ME, STAR*D Study Team. Bupropion-SR, sertraline, or venlafaxine-XR after failure of SSRIs for depression. N Engl J Med 2006 Mar 23;354(12):1231-1242. [doi: 10.1056/NEJMoa052963] [Medline: 16554525 ]

50. Bang H, Ni L, Davis CE. Assessment of blinding in clinical trials. Control Clin Trials 2004 Apr;25(2):143-156. [doi: 10.1016/j.cct.2003.10.016] [Medline: 15020033]

51. Bang H. Random guess and wishful thinking are the best blinding scenarios. Contemp Clin Trials Commun 2016 Aug 15;3:117-121. [doi: 10.1016/j.conctc.2016.05.003] [Medline: 27822568]

52. Turner EH, Matthews AM, Linardatos E, Tell RA, Rosenthal R. Selective publication of antidepressant trials and its influence on apparent efficacy. N Engl J Med 2008 Jan 17;358(3):252-260. [doi: 10.1056/NEJMsa065779] [Medline: 18199864]

53. Hepner KA, Greenwood GL, Azocar F, Miranda J, Burnam MA. Usual care psychotherapy for depression in a large managed behavioral health organization. Adm Policy Ment Health 2010 May;37(3):270-278. [doi: 10.1007/s10488-009-0247-6] [Medline: 19859800]

54. Furukawa TA, Noma H, Caldwell DM, Honyashiki M, Shinohara K, Imai H, et al. Waiting list may be a nocebo condition in psychotherapy trials: a contribution from network meta-analysis. Acta Psychiatr Scand 2014 Sep;130(3):181-192. [doi: 10.1111/acps.12275] [Medline: 24697518]

\section{Abbreviations}

BDI-II: Beck Depression Inventory-II

CBT: cognitive behavioral therapy

FIBSER: Frequency, Intensity, and Burden of Side Effects Ratings

PHQ-9: Patient Health Questionnaire 9

RCT: randomized controlled trial

Edited by G Eysenbach; submitted 29.07.17; peer-reviewed by K Hallgren, S Moritz; comments to author $24.08 .17 ;$ revised version
received 02.09.17; accepted 23.09.17; published 03.11.17
Please cite as:
Mantani A, Kato T, Furukawa TA, Horikoshi M, Imai H, Hiroe T, Chino B, Funayama T, Yonemoto N, Zhou Q, Kawanishi N
Smartphone Cognitive Behavioral Therapy as an Adjunct to Pharmacotherapy for Refractory Depression: Randomized Controlled
Trial
J Med Internet Res 2017;19(11):e373
URL: $\underline{\text { http://www.jmir.org/2017/11/e373/ }}$
doi: $\underline{10.2196 / j m i r .8602}$
PMID: $\underline{29101095}$

(CAkio Mantani, Tadashi Kato, Toshi A Furukawa, Masaru Horikoshi, Hissei Imai, Takahiro Hiroe, Bun Chino, Tadashi Funayama, Naohiro Yonemoto, Qi Zhou, Nao Kawanishi. Originally published in the Journal of Medical Internet Research 
(http://www.jmir.org), 03.11.2017. This is an open-access article distributed under the terms of the Creative Commons Attribution License (https://creativecommons.org/licenses/by/4.0/), which permits unrestricted use, distribution, and reproduction in any medium, provided the original work, first published in the Journal of Medical Internet Research, is properly cited. The complete bibliographic information, a link to the original publication on http://www.jmir.org/, as well as this copyright and license information must be included. 